\title{
A tensorial description of stresses in triphasic granular materials with interfaces
}

\author{
R. Wan", J. Duriez ${ }^{\mathrm{a}}$, F. Darve ${ }^{\mathrm{b}}$ \\ ${ }^{a}$ Department of Civil Engineering, University of Calgary, Calgary, AB, T2N 1N4, Canada \\ ${ }^{b}$ Univ. Grenoble Alpes, 3SR, F-38000 Grenoble, France \\ CNRS, 3SR, F-38000 Grenoble, France
}

\begin{abstract}
The description of stress transmission in an unsaturated granular material in the low water saturation range (pendular regime) is studied within the micromechanics of a three-phase medium. Considering an REV (Representative Element Volume) comprised of solid grains and isolated inviscid water menisci whose statistical distributions are known, micro- and macro-relationships can be derived using a volume-averaging of stress for each one of the phases in a manner analogous to the derivation of Love-Weber equation defining stress in a dry granular medium. The main difference with respect to previous works in the literature lies in the homogenization technique whereby singular surfaces such as an air-water interface exhibiting surface tension are treated as an additional phase for which a so-called membrane stress is defined. Well-known contractile skin effects and the anisotropy of capillary stresses in unsaturated soils can be formally identified in the proposed tensorial expression that encompasses the statistics of not only grain contact normals, but also that of menisci spatial orientations and their surfaces, including water saturation. Solid grains are considered strictly incompressible with water being also an incompressible and inviscid liquid. An effective stress tensor that controls the strength of unsaturated granular media is hence proposed and verified based on discrete element modelling (DEM) numerical simulations of triaxial compression and simple shear tests on pendular-state granular soils at different confining pressures and matric suctions. The non-sphericity of the so-called capillary stress is also numerically demonstrated.
\end{abstract}

Keywords: pendular regime, effective stress, interfacial tension, wetting-drying, suction 


\section{1}

2

,

,

\section{Introduction}

The derivation of a tensorial stress field in a continuum representation of an unsaturated particulate medium like a geomaterial is well studied in the literature; see [33, 13, 26], among others. While microstructural aspects have lately been subject of considerable interest, a largely theoretical issue that is often evoked is the definition of a single-valued effective stress $[8,32,42]$ that controls both deformation and strength in such unsaturated media, and which replaces the celebrated Terzaghi's effective stress in the fully saturated case. This question takes on a more practical importance in geotechnical/geo-environmental engineering, motivated by the shrinkage, swelling, yielding and collapse of unsaturated soils at low water saturation upon drying and wetting cycles associated with weather change or the natural hydrologic cycle, e.g. [29].

Herein, we are particularly interested in the pendular regime where water saturation is low and the water phase is held in between the interstices of the granular material (soil) in the form of isolated menisci [4, 38]. In this case, the solid phase is comprised of a grain skeleton whose interstices are occupied by air as a continuous phase and water as a discontinuous phase, with interfaces that separate the various phases. Hence, the mechanics of the unsaturated granular system is invariably governed by the different phases whose individual behaviours are well defined, and also interfaces that are endowed with thermodynamics properties $[20,30]$. Problems associated with unsaturated soil mechanics and related 
fields specifically involve fluid flow, stress, thermal, chemical and deformation phenomena which are essentially coupled. Here in this paper, we are specifically interested in stress phenomena that encompass classical geotechnical problems under unsaturated conditions where the question of stress transport and strength are of critical concern, but yet difficult to discern.

Historically, Bishop's stress [5] as an extension to Terzaghi's effective stress has been a commonly used stress in unsaturated soils according to the following tensorial form:

$$
\begin{aligned}
\sigma_{i j}^{\prime} & =\sigma_{i j}-(1-\chi) u_{a} \delta_{i j}-\chi u_{w} \delta_{i j} \\
& =\bar{\sigma}_{i j}+\chi\left(u_{a}-u_{w}\right) \delta_{i j}
\end{aligned}
$$

where $\sigma_{i j}^{\prime}$ is the Bishop's stress tensor, $\bar{\sigma}_{i j}=\left(\sigma_{i j}-u_{a} \delta_{i j}\right)$ is the so-called net stress, $\chi$ is generally assumed to be a function of degree of saturation and is zero for dry soil and unity for saturated soil, and the term $\left(u_{a}-\right.$ $\left.u_{w}\right)$ represents matric suction $s$. Herein, soil mechanics sign convention is adopted throughout the paper, i.e. compressive stresses and strains are positive.

Over the years, Bishop's equation has been the subject of controversy [23] and even Bishop and Blight [6] admitted that there are uncertainties over the definition of parameter $\chi$, although much later on, Gray and Hassanizadeh [18] proved that Bishop's stress is thermodynamically consistent when $\chi$ is associated with degree of saturation. Moreover, Blight [7] 
argued that the value of $\chi$ depends on stress and strain levels; whereas nowadays, Wan et al. [44], Duriez and Wan [15] have established its link to fabric and meniscus spatial distribution which furthermore destroys the isotropic character of the matric suction in the original Bishop's equation.

Uncertainties in finding a single-valued stress variable prompted the idea that the behaviour of unsaturated soils should be governed independently by two variables: the net stress $\left(\sigma-u_{a}\right)$ and matric suction $\left(u_{a}-u_{w}\right)$. Fredlund and Morgenstern [17] went on further to suggest that any two of the stress state variables $\bar{\sigma}=\left(\sigma-u_{a}\right), \sigma^{\prime}=\left(\sigma-u_{w}\right)$, and $s=\left(u_{a}-u_{w}\right)$ could be employed. Furthermore, Houlsby [21] developed the concept of power input into unsaturated soils, and hence introduced the notion of work conjugate stress and strain increment variables for constitutive modelling, but without including the energy of the interfaces. The idea of average soil skeleton thus emerged.

More recent studies have focused on the understanding of unsaturated soil behaviour through continuum approaches such as mixture theory [9, 40]. As such, there has been a number of stress measures defined for unsaturated granular media and which have made their way to soil mechanics and reservoir engineering. These are the total stress, the skeleton stress [19], the net stress [1], the generalized Bishop [5] stress, and the Skempton [41] stress. Approaches that are based on total stresses have even been proposed for triphasic media, but then, they would necessitate the writing of equations of equilibrium and associated boundary conditions for 
each phase; see [30] which circumvents this latter requirement. Given the overall progress of granular mechanics in the past several decades, renewed efforts to address the above theoretical and practical questions seem appropriate. With this motivation, this paper provides a systematic microstructural study in which a tensorial stress expression is derived for a triphasic material composed of air, water and solid, with the water phase being discontinuously distributed as isolated menisci (liquid bridges) between particles that can be either in contact or not. As for the solid phase, we consider distinct idealized particles of spherical shape. Additionally, the air-water interface along with a jump condition at the common line is included in the derivation as a fourth phase .

The proposed method uses a volume averaging of stress for each one of the phases in a manner analogous to the derivation of Love-Weber equation [28, 45] defining stress in a dry granular medium to arrive at a tensorial expression for stress that encompasses the statistics of not only grain contact normals, but also that of menisci spatial orientations and menisci surfaces, including water saturation. More importantly, an effective stress tensor can be recognized as the difference between the total stress tensor and so-called capillary stresses that include contributions from suction, surface tension, and air-water interface associated tensors. As new results, we identify menisci induced effects within the unsaturated granular medium through:

1. a surface based fabric tensor of menisci scaled by the matric suction, 


\subsection{Preliminaries}

The notion of stress within a continuous body goes as far back to Cauchy who introduced this abstract conceptual scheme where the mutual action of two bodies in contact, or two parts of the same body separated by an imaginary surface is contemplated [28]. This powerful conceptual idea can be extended to the definition of a stress tensor under quasi-static conditions for a heterogeneous body consisting of discrete particles of volume $V_{p}$. These are considered rigid, endowed with stress $\sigma^{p}$, and interact through contact points $c$ with pairwise interparticle force $\mathbf{f}^{p}$ in a granular assembly of volume $V$. Thus, the celebrated Love-Weber formula gives:

$$
\begin{aligned}
\sigma & =\frac{1}{V} \sum_{p} \int_{\partial V_{p}}\left(\sigma^{p} \cdot \mathbf{n}\right) \otimes \mathbf{x}^{p} d S \\
& =\frac{1}{V} \sum_{p} \sum_{c} \mathbf{f}^{p} \otimes \mathbf{x}^{p}
\end{aligned}
$$


where $\left(\sigma^{p} \cdot \mathbf{n}\right)$ is the traction vector on the particle $p$ with outward normal $\mathbf{n}$, and the vector $\mathbf{x}^{p}$ represents the spatial position of applied traction with respect to an arbitrary origin.

When sweeping over all contacts for every particle in volume $V$ and by expressing the position vector $\mathbf{x}^{p}$ as a function of the particle's centroid and radius vector, we get the classic expression given by:

$$
\sigma=\frac{1}{V} \sum_{\alpha \beta} \mathbf{f}^{\alpha \beta} \otimes \boldsymbol{\ell}^{\alpha \beta}
$$

where the summation is carried out over distinct pairs of particles $\alpha \beta$ in $V, \mathbf{f}^{\alpha \beta}$ and $\ell^{\alpha \beta}$ denote, respectively, the pairwise interaction force and branch vector giving distance of separation.

Equation (3), derived with the assumption of a quasi-static medium in the absence of any body forces, serves as the basis for calculating volumeaverage stresses in heterogenous media. For the case where the discrete particles are embedded into a matrix which includes singular surfaces over which surface tension occurs, the integral term in Eq. (2a) has to be amended. This is because some components of the stress tensor suffer a discontinuity associated with surface tension, as will be seen next when calculating the volume-average stress in a triphasic medium.

\subsection{Averaging process with stress discontinuities}

Consider a representative element volume (REV) of volume $V$ containing a statistically significant number of particles and water menisci as illustrated in Fig. 1 . 


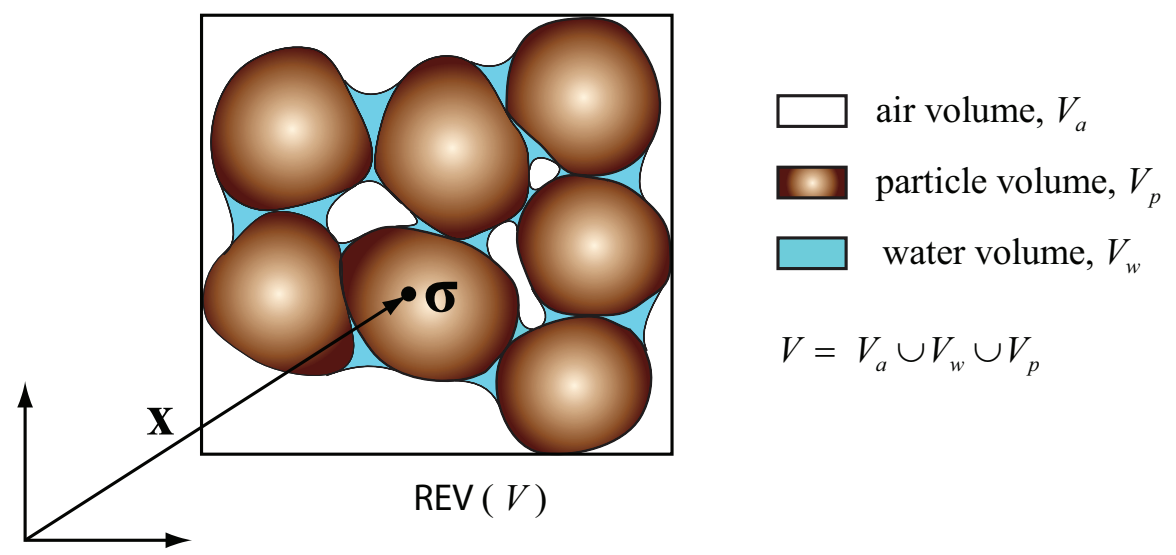

Figure 1: REV of pendular unsaturated medium

It also represents an averaging volume whose dimensions are large compared with the characteristic microscale (particle and water meniscus sizes) of the unsaturated granular material, but still arbitrarily small compared to the dimension of the soil domain. The solid particles are assumed to be strictly rigid interacting with incompressible air and inviscid water.

In practical terms, we wish to know what stress is generated within the REV when loads (mechanical or hydraulic) are applied on its external boundary. Since the actual stress $\sigma_{i j}$ varies with position $\mathbf{x}$ in the REV, the 'bulk stress' can be defined from an ensemble (volume) averaging. However, a closer look at the REV reveals that we are faced with a three-phase system composed of solid (s), air (a) and water (w) separated by interfacial surfaces upon which a singularity in the stress distribution exists due to surface tension. A volume integral of the stress over such a region is then strictly speaking improper, and must be thus interpreted appropriately. 
As such, the average stress $\Sigma_{i j}$ is computed just as volume averages of stress in all phases concerned, including the interfaces, i.e.

$$
\Sigma_{i j}=\frac{1}{V}\left\{\int_{V^{p}} \sigma_{i j} d V+\int_{V^{w}} \sigma_{i j} d V+\int_{V^{a}} \sigma_{i j} d V+\int_{V^{i n t}} \sigma_{i j} d V\right\}
$$

Herein, it is appropriate to regard the interface between air and water as a layer of small thickness $\epsilon$, and to subsequently take the limit $\epsilon \rightarrow 0$. For a given point on the interface, if the local normal is denoted by unit normal $\mathbf{n}$, then the surface tension force field tensor can be written in the form $\gamma\left(\delta_{i j}-n_{i} n_{j}\right)$ where $\gamma$ is the coefficient of surface tension. On the other hand, the stress components along directions in the tangent plane of the air-water interfacial surface are very large in the interface layer. Therefore, the correct stress integral properties can be obtained by taking the singular part of the stress tensor in the interface layer to be in the form $\sigma_{i j}=\gamma\left(\delta_{i j}-\right.$ $\left.n_{i} n_{j}\right) / \epsilon$.

Noting the above, the contribution of the volume integral in Eq. (4) from the portion of the interfacial surface $S^{a w}$ lying within the REV is then

$$
\lim _{\epsilon \rightarrow 0} \int_{V_{\text {int }}} \sigma_{i j} d V=\int_{S^{a w}} \gamma\left(\delta_{i j}-n_{i} n_{j}\right) d S
$$

The integral on the right hand side of Eq. (5) can be alternatively written as

$$
\int_{\text {Saw }} \gamma\left(\delta_{i j}-n_{i} n_{j}\right) d S=\int_{\text {Saw }} \gamma n_{i} \operatorname{div} \mathbf{n} x_{j} d S
$$

by applying Stokes's theorem to the quantity $\epsilon_{k i l} x_{i} n_{l}$, regarded as a vector with components given by the different values of $k$ [36]. 
The term, div $\mathbf{n}$, is in fact the curvature of the interficial surface containing the local normal $\mathbf{n}$, and hence $\left(\gamma n_{i} \operatorname{div} \mathbf{n}\right)$, which is the normal curvature force per unit area, represents the jump in the normal stress across the air-water interface due to surface tension, i.e. $\left(u_{a}-u_{w}\right) n_{i}$.

Noting the above and considering the pressures of the water and air phases to be hydrostatic, the volume-average stress in Eq. (4) can be rewritten as:

$$
\begin{aligned}
\Sigma_{i j} & =\frac{1}{V} \sum_{p} \int_{V^{p}} \sigma_{i j} d V+\frac{1}{V} \int_{V^{w}} u_{w} \delta_{i j} d V+\frac{1}{V} \int_{V^{a}} u_{a} \delta_{i j} d V \\
& +\frac{1}{V} \sum_{l} \int_{S^{a w}} \gamma n_{i} \operatorname{div} \mathbf{n} x_{j} d S
\end{aligned}
$$

where $p$ and $l$ are the number of particles and liquid bridges respectively, $V^{a}$ and $V^{w}$ are the volume occupied by air and water phases respectively, while $u_{a}$ and $u_{w}$ are the air and water pressures respectively.

Applying the divergence theorem to the volume integral concerning each particle in Eq. (7) leads to a surface integral just like in the opening Eq. (2a), except that the tractions exerted on the particle's surface with outward normal $\mathbf{n}$ have now various origins. For instance, we will find contributions from pair-wise particle contact forces due to external loading, as well as actions of air and water pressures on $\operatorname{dry}\left(\partial V_{p}^{a}\right)$ and wetted surfaces $\left(\partial V_{p}^{w}\right)$ respectively. Based on the above and noting Eq. (3), it 
follows that

$$
\begin{aligned}
\Sigma_{i j} & =\frac{1}{V} \sum_{\alpha \beta} f_{i}^{\alpha \beta} \ell_{j}^{\alpha \beta}+\frac{u_{w}}{V} \sum_{p} \sum_{l} \int_{\partial V_{p}^{w}} n_{i} x_{j} d S+\frac{u_{a}}{V} \sum_{p} \int_{\partial V_{p}^{a}} n_{i} x_{j} d S \\
& +\frac{V_{w}}{V} u_{w} \delta_{i j}+\frac{V_{a}}{V} u_{a} \delta_{i j}+\frac{1}{V} \sum_{l} \int_{S_{a w}} \gamma n_{i} \operatorname{div} \mathbf{n} x_{j} d S
\end{aligned}
$$

where tractions acting on vanishing surface area over the contour $\partial V_{p}$ of each particle $p$ have been transformed into point (contact) forces $f_{i}^{\alpha \beta}$ between pairs of particles $\alpha \beta$ joined by branch vector $\ell_{j}^{\alpha \beta}$.

By applying Stokes's theorem (see Appendix A), the interface integral can be re-written as

$$
\frac{1}{V} \int_{S^{a w}} \gamma n_{i} \operatorname{div} \mathbf{n} x_{j} d S=-\frac{1}{V} \int_{\mathcal{C}} T_{i}^{a w} x_{j} d \ell+\frac{1}{V} \int_{S} \frac{\partial \gamma}{\partial x_{i}} x_{j} d S
$$

where $T_{i}^{a w}=\gamma t_{i}^{a z w}$ refers to the surface tension force acting at the intersection of the liquid bridge and the particle, i.e. the interface solid-air-water which creates the so-called contractile skin within the unsaturated granular assembly.

Furthermore, noting that the surface area of a particle $\partial V_{p}$ can be decomposed as a union of dry (air) surfaces $\partial V_{p}^{a}$ and wetted surfaces over $l$ liquid bridges, i.e. $\partial V_{p}=\partial V_{p}^{a} \cup \partial V_{p}^{w}$, the surface integral over the dry surfaces of a particle can be written as:

$$
\int_{\partial V_{p}^{a}} n_{i} x_{j} d S=\int_{\partial V_{p}} n_{i} x_{j} d S-\sum_{l} \int_{\partial V_{p}^{w}} n_{i} x_{j} d S
$$

Finally, in the absence of any gradient in surface tension force $\left(\partial \gamma / \partial x_{i}=\right.$ 
0) we get:

$$
\begin{aligned}
\Sigma_{i j} & =\frac{1}{V} \sum_{\alpha \beta} f_{i}^{\alpha \beta} \ell_{j}^{\alpha \beta}-\frac{\left(u_{a}-u_{w}\right)}{V} \sum_{p} \sum_{l} \int_{\partial V_{p}^{w}} n_{i} x_{j} d S \\
& +\frac{u_{a}}{V} \sum_{p} \int_{\partial V_{p}} n_{i} x_{j} d S+\frac{V_{w}}{V} u_{w} \delta_{i j}+\frac{V_{a}}{V} u_{a} \delta_{i j} \\
& -\frac{1}{V} \sum_{p} \sum_{l} \int_{\mathcal{C}} T_{i}^{a w} x_{j} d l
\end{aligned}
$$

For a granular system consisting of particles of radius $R_{p}, x_{i}=R_{p} n_{i}$, and noting $\phi=\left(V_{a}+V_{w}\right) / V$ and $S_{r}=V_{w} /\left(V_{a}+V_{w}\right)$, with $V=V_{s}+$ $V_{w}+V_{a}$, it follows after some manipulations that:

$$
\Sigma_{i j}=u_{a} \delta_{i j}+\frac{1}{V} \sum_{\alpha \beta} f_{i}^{\alpha \beta} \ell_{j}^{\alpha \beta}-\chi_{i j}\left(u_{a}-u_{w}\right)-B_{i j}
$$

where

$$
\begin{aligned}
\chi_{i j} & =\frac{1}{V} \sum_{p} R_{p} \sum_{l} \int_{\partial V_{p}^{w}} n_{i} n_{j} d S+\phi S_{r} \delta_{i j} \\
B_{i j} & =\frac{1}{V} \sum_{p} R_{p} \sum_{l} \int_{\mathcal{C}} T_{i}^{a w} n_{j} d l
\end{aligned}
$$

Equation (12) serves to establish an equivalence between a non homogeneous granular system with discontinuous air-water interfaces and an equivalent homogeneous REV through the volume-average stress tensor $\Sigma$. In fact, this relation bears some resemblance to Bishop's equation, but is more complex in three major distinct aspects. First, the matric suction is no longer an isotropic quantity, but is now governed by a Bishop-like parameter which is a tensor $\chi$ whose explicit form is known with dependences 
on liquid bridge spatial distribution and degree of saturation, among others. Second, the influence of air-water interfacial tension on the granular system behaviour is included through the new term $\boldsymbol{B}$ which involves integration of lineal surface tension forces over contours defined by the wetting of liquid bridges with the solid particles. Third, the expression makes a clear distinction between point forces at particle contacts and lineal forces arising from surface tension. In fact, these point forces are exerted at contacts of particle pairs $\alpha \beta$ to give rise to a so-called contact stress tensor $\sigma^{\text {cont }}$ through the Love-Weber formula which is clearly recognizable in the first line of Eq. (12).

To make particular reference to the liquid bridge contributions to the total stress $\Sigma$, we conveniently define a so-called capillary stress tensor taken to be:

$$
\sigma^{\text {cap }}=-(s \chi+B)=\Sigma-u_{a} \delta-\sigma^{\text {cont }}
$$

Note that the "capillary stress" terminology, also used elsewhere in [39], is equivalent to the "suction stresses" alluded in Lu and Likos [29].

We will next endeavour to investigate the nature of capillary stresses $\sigma^{\text {cap }}$, as well as check whether the contact stress $\sigma^{\text {cont }}$ can be regarded as an effective stress in Terzaghi's sense. To achieve such a goal, we will adopt a DEM calculation approach to determine the water phase distribution within an unsaturated granular system so that Eq. (12), the central piece of this study, can be formally evaluated. 


\section{Discrete modelling of a triphasic medium}

\subsection{The discrete model}

Generally speaking, numerical simulation of the mechanics of granular material based on DEM consists of calculating the motion of a collection of particles, the so-called discrete elements (DE), under the action of solely point forces. Deformation of the granular ensemble then comes out of the relative displacements of particles calculated following Newton's law of motion under interaction forces and torques. The numerical simulations can describe quite accurately salient behavioural features of real granular materials, although particle shapes are idealized as disks or spheres and the number of particles is relatively small compared to actual granular masses.

For the discrete modelling of granular multiphasic systems, liquid and gas phases are included through the forces they impose on solid particles as described in [35, 39, 31] for the unsaturated case, and in [11] for the fully saturated case. It is worth noting that an embedded flow model [12] is used in [11] to compute spatially varying water pressure field. However, if an homogeneous distribution of matric suction is assumed within an unsaturated granular material, the water phase characteristics can be readily computed. It is under such an assumption that the liquid bridge distribution specific to the pendular regime can be determined by solving Laplace's equation [25] for all particle pairs. Following this approach, Scholtès et al. [39] proposed a discrete model for unsaturated conditions, 
within Yade open source code [43]. Therefore, the characteristics of liquid menisci being determined from Laplace's equation for a zero contact angle, so-called capillary forces due to the water and air phases can be readily determined from the radius $R$ of the particle, surface tension $\gamma$, matric suction $s$, and filling angle $\alpha$ (Fig. 2), i.e.

$$
\mathbf{f}^{\text {cap }}=\pi R \sin ^{2} \alpha(2 \gamma+R s) \mathbf{x}
$$

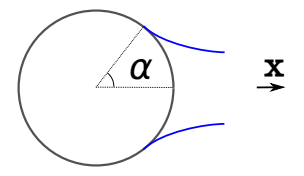

Figure 2: Liquid bridge geometry

Other discrete models for unsaturated granular materials differ mainly in the way the capillary force is computed, using often approximations based on assumptions made for the liquid volume [35, 31].

In this study, we use the discrete model proposed by Scholtès et al. [39] which is already implemented in the open source computer code YADE. In addition to the capillary forces, contact interaction forces are computed from three basic numerical parameters and particles relative displacements. Classically, the contact behavior is elastic in the normal direction (tensile states being ruled out), and elastic-plastic in the tangential direction. Two first parameters, namely $Y$ and $P$, refer to the normal and tangential con- 
tact stiffnesses $k_{n}, k_{t}$ for an interaction between particles $A$ and $B$ :

$$
\begin{aligned}
& k_{n}=\frac{2 Y R_{A} R_{B}}{R_{A}+R_{B}} \\
& k_{t}=P k_{n}
\end{aligned}
$$

Expressing $k_{n}$ and $k_{t}$ according to the particles radii $R_{A}$ and $R_{B}$ leads to a size independent behavior of the model in dry conditions. The third contact parameter is the local contact friction angle $\varphi$ that restricts the tangential force: $\left\|\vec{F}_{t}\right\| \leqslant F_{n} \tan (\varphi)$. A fourth numerical parameter enters as the particle size as discussed in Duriez and Wan [16] where the capillary force computations between contacting and distant particles make the response of the unsaturated granular system particle size dependent.

We herein choose a granular ensemble with a uniform particle size distribution whose characteristics appear in Table1 with other model parameters.

\begin{tabular}{|c|c|c|c|c|}
\hline $\begin{array}{c}Y \\
(\mathrm{MPa})\end{array}$ & $\begin{array}{c}P \\
(-)\end{array}$ & $\begin{array}{c}\varphi \\
\left({ }^{\circ}\right)\end{array}$ & $\begin{array}{c}D_{\max } \\
D_{\min }\end{array}$ & $\begin{array}{c}D_{50} \\
(\mathrm{~mm})\end{array}$ \\
\hline 50 & 0.5 & 30 & 3 & 0.04 \\
\hline
\end{tabular}

Table 1: Model parameters

The packing procedure used to generate the granular assembly involves the isotropic compression of an initial particles gas with the desired particle size distribution. During this compression, the local contact friction angle is very low. The procedure outputs a sample with an isotropic fabric, a mean coordination number of 4.8 and a low porosity $n \approx 0.369$, under 1 
$\mathrm{kPa}$ of isotropic pressure. On the other hand, the confining pressures considered here in the shearing phase range in the tens of $\mathrm{kPa}$ and the DEM sample displays a typically dense behaviour [15].

\subsection{Hydraulic behaviour of the discrete model}

In the pursuit of fundamentals, the wetting and drying of a granular material is examined through DEM and provides a framework within which we can verify the proposed stress equation derived from homogenization.

Computing the water phase distribution, the discrete model allows us to readily construct the Soil-Water Characteristic Curves (SWCC) numerically. Hydraulic hysteresis is herein introduced through different liquid bridge assumptions. As water vapour condensates primarly over solid surfaces, initial wetting paths are simulated creating liquid bridges between contacting particles only. On the other hand, drying path simulations involve liquid bridges between distant particles as well as contacting particles, with the only condition being the existence of a solution to Laplace's equation.

Fig. 3 depicts the drying and wetting SWCC of the discrete model together with some experimental data point for an Ottawa sand with a mean diameter $D_{\text {mean }} \approx 0.172 \mathrm{~mm}$ [27].

A limited hysteresis between drying and wetting is simulated because known hysteresis mechanisms are not included in the numerical model. First, the same contact angle value is considered upon drying and wetting. 


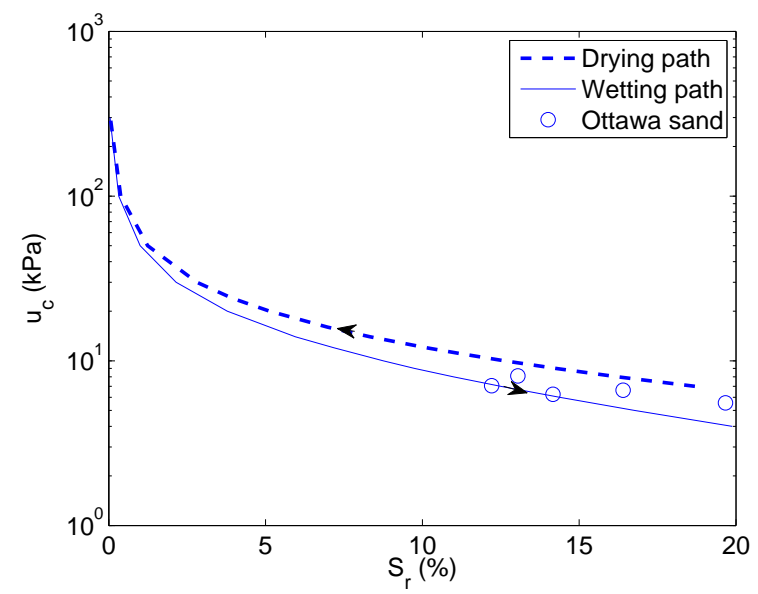

Figure 3: SWCCs of the discrete model. Ottawa sand data from [27]

Second, liquid bridges are uniformly distributed according to uniform matric suction and particles distances and radii. As such, the control that smaller pores exert on the filling of larger pores according to the hydraulic loading path - the so-called ink-bottle effects - cannot be reproduced. Simulation of such effects would certainly require the actual calculation of the flow of the two fluid phases inside the pore space, which is outside the scope of this paper.

\section{Discussion of discrete modelling for multiphasic media}

DEM may be considered as a numerical homogenization approach, where the stress tensor for a DEM sample arises from the interaction forces between discrete elements. However, when applied to multiphasic materials, we argued in another work [16] that DEM provides an inconsistent average stress description, especially in the case where mechanical 
actions corresponding to the fluid phases deviate significantly from being point forces. This inconsistency arises from the fact that actions of the fluid phases on the solid phase are replaced by a resultant force, ignoring the nature of their spatial distribution. Indeed, our volume-average stress equation (12) reveals lineal forces due to surface tension and surface forces due to fluid pressures, besides point forces at particle contacts. The above important issue is next examined in greater details.

\subsection{Biphasic case}

For the fully saturated case involving a uniform water pressure $u_{w}$ within the pore space, replacing the action of liquid phase on the solid phase with resultant forces would lead to the following within DEM calculations:

- boundary DE (such as rigid platens) would sustain added forces $\mathbf{f}^{w}=-u_{w} S \mathbf{n}$, with $S$ the adequate surface and $\mathbf{n}$ the inwards external normal,

- no extra forces would be imposed on any other DE since the water phase induces no resultant forces on the solid phase in this case.

As such, total stresses defined from the resulting forces acting on boundary DE are consistent with applied external loads. However, homogenization of the forces interior to the discrete model towards these total stresses is impossible, since the interior forces reflect the skeleton behaviour only, corresponding to Terzaghi effective stress [11]. 


\subsection{Triphasic case}

As for the present unsaturated discrete model, the contact and capillary forces interior to the model may be exactly homogenized to the total stresses acting along the boundaries [39, 16], showing an additivity qualitatively similar to the one of the homogenization approach presented Section 2.2. In the end, the total stress $\Sigma$ for the unsaturated discrete model is the sum of $\sigma^{\text {cont }}$, the same term as within the homogenization approach, function of contact forces, and another stress tensor denoted again as a capillary stress tensor $\sigma^{\text {cap }}=\Sigma-\sigma^{\text {cont }}$.

Within DEM, this capillary stress tensor is computed from the capillary forces exerted by the fluid phases on the solid phase. However, fluid phases are not simulated themselves, and point forces consideration does not conform with both surface and lineal characters of the mechanical actions (fluid pressures and surface tension forces) exerted by the fluids on the solid. For these reasons, the total stresses description of an unsaturated soil as provided by the discrete model is not consistent with the averaging approach of Section 2.2 in the general case. In fact, both approaches are consistent when liquid volume and wetted surfaces as well as contours tend to vanish, which is the case with a very good approximation for very low degrees of saturation (few percents); otherwise substantial differences appear [16].

To illustrate here this point, hydraulic loadings are imposed to the discrete model. Wetting and drying paths are simulated by varying the ma- 
tric suction under an isotropic stress state $p=10 \mathrm{kPa}$ kept constant on the boundaries.

First, capillary stresses as described by the DEM, denoted $\sigma_{\mathrm{DEM}}^{\mathrm{cap}}$, are directly computed from capillary forces [39, 16]:

$$
\sigma_{\mathrm{DEM}}^{\mathrm{cap}}=\frac{1}{V} \sum_{l} \mathbf{f}_{\beta}^{\mathrm{cap}} \otimes \boldsymbol{\ell}_{\alpha \beta}
$$

Equation (16) considers all particles pairs $\alpha \beta$ linked by a liquid bridge $l$, with $\mathbf{f}_{\beta}^{\text {cap }}$ the capillary force as sustained by $\beta$, and $\ell_{\alpha \beta}$ the so-called branch vector from the center of $\alpha$ to the center of $\beta$ as defined earlier in the paper. Second, we take advantage of the output the discrete model gives such as features of the liquid phase (filling angles $\alpha$, menisci volumes) as it would exist in the simulated unsaturated soil. Hence, another capillary stress tensor $\sigma_{\text {hom }}^{\text {cap }}$ is computed using the homogenization formula (Eq. 12):

$$
\sigma_{\text {hom }}^{\text {cap }}=-s \chi-B
$$

During hydraulic loading, both tensors $\sigma_{\mathrm{DEM}}^{\text {cap }}$ and $\sigma_{\text {hom }}^{\text {cap }}$ turn out to be spherical (isotropic), with $B=0$ with regard to $\sigma_{\text {hom }}^{\text {cap }}$. Indeed, the packing of the DEM sample is here isotropic leading to an isotropic liquid bridge distribution, irrespective of whether a meniscus exists between contacting particles only (along the wetting path) or not (along the drying path). Taking advantage of the sphericity of both capillary stress tensors in the above, the mean capillary pressures $p_{\mathrm{DEM}}^{\mathrm{cap}}=1 / 3 \operatorname{tr}\left(\sigma_{\mathrm{DEM}}^{\mathrm{cap}}\right)$ and $p_{\text {hom }}^{\text {cap }}=1 / 3 \operatorname{tr}\left(\sigma_{\text {hom }}^{\text {cap }}\right)$ are compared. 
The discrepancies between $p_{\text {DEM }}^{\text {cap }}$ and $p_{\text {hom }}^{\text {cap }}$ mentionned earlier appear in Fig. 4. As in [16], the DEM model overestimates (in absolute value) the mean capillary pressure in the general case. For a common value at very low water contents $\left(S_{r} \rightarrow 0\right)$, when both approaches are equivalent, $p_{\text {DEM }}^{\text {cap }}$ deviates more and more from $p_{\text {hom }}^{\text {cap }}$ up to $40-50 \%$ for $S_{r}=15 \%$. It is seen that this discrepancy increases according to the wetted surfaces which the discrete model does not directly take into account, contrary to the homogenization approach (Fig. (5). While qualitative trends are similar along wetting, influences of $S_{r}$ on $p_{\mathrm{DEM}}^{\text {cap }}$ and $p_{\mathrm{hom}}^{\text {cap }}$ are even different along the drying path. This corresponds to greater wetted surfaces along drying rather than wetting (Fig. 5).

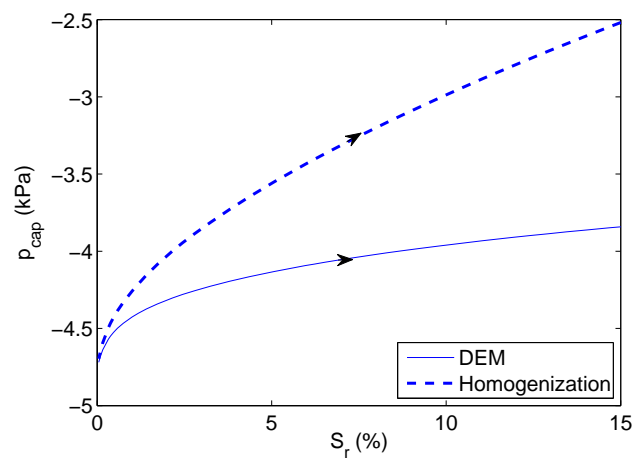

(a) Wetting

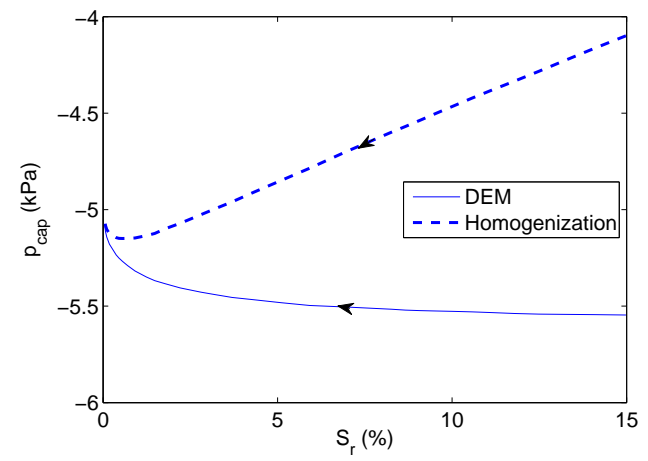

(b) Drying

Figure 4: Capillary pressure from DEM or homogenization approach along hydraulic loadings (for both approaches $p^{\text {cap }}$ is discontinuous at the origin, with $p^{\text {cap }}\left(S_{r}=0\right)=0$, not represented)

It is interesting to note that the higher wetted surfaces developed along the drying path than along the wetting path come from a higher number of 


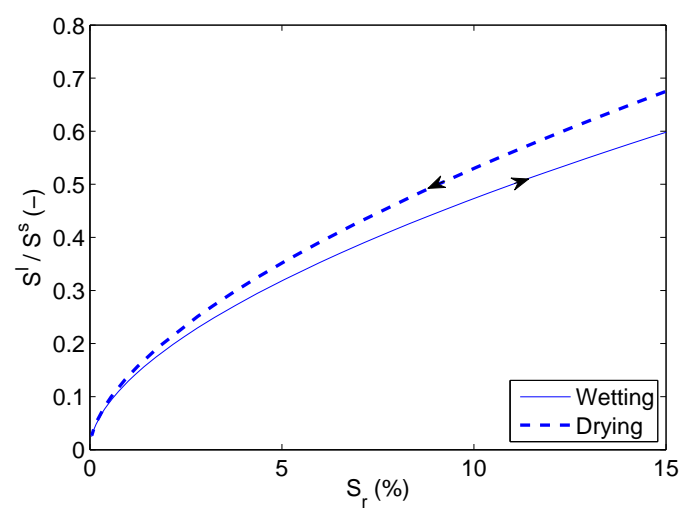

Figure 5: Solid surfaces wetted by the liquid, $S^{l}$, respective to whole solid surface $S^{s}$

liquid bridges. For a degree of saturation of $10 \%$ for instance, considering a drying path leads to $50 \%$ more liquid bridges between both distant and touching particles when compared with wetting. The average volume of these liquid bridges is consistently one third smaller, however each liquid bridge extends roughly along the same solid surface for both hydraulic loading paths. For these configurations, the average filling angle is $30.5^{\circ}$ along drying, and $34.8^{\circ}$ along wetting.

It is worth noticing that classical ("dry") DEM simulations also encompass the current discussion of the validity of this method if actual physical forces are distributed. Indeed, the use of resultant forces is arguably presented in [3] as adequate when contact areas (in reality), i.e. overlaps (in DEM models), are negligible-which is a classical assumption for the validity of DEM [14, 34, 37, 22]. As for the homogenization approach, this assumption enters directly into the computations, so that $\int \sigma \mathbf{n} \otimes \mathbf{x} d S$ can be replaced by $\sum \mathbf{f} \otimes \mathbf{x}[2]$. 
Finally, we wish to conclude from these results that DEM applied to multiphasic materials provides in some cases an inconsistent description of the average, or total, stresses. Obviously, such description is in fact not relevant for saturated cases for which the behaviour is known to be governed by Terzaghi's effective stress, provided that both fluid and solid phases are incompressible. However, it is in our opinion that searching for a single-valued effective stress for the unsaturated case through numerical experiments, total stresses have first to be adequately simulated.

\section{Hydraulic loading}

We focus now on the capillary stress tensor, as computed from the homogenization approach. First, we consider the same hydraulic loading paths as in Section 4.2. Regarding the discussion on the comparison between DEM and the homogenization approach, only wetting paths are considered, up to a maximum degree of saturation $S_{r}=10 \%$, so that DEM and the homogenization approach are consistent, qualitatively and to some extent quantitatively.

\subsection{Isotropic packings}

The initial isotropic packing of the sample confers an isotropy to the liquid phase, given that liquid bridges appear at every geometrical contacts. As mentioned earlier, this makes the capillary stress tensor to be spherical, with $\sigma^{\text {cap }}=-s \chi$ since $\boldsymbol{B}=\mathbf{0}$ for isotropic packings, under a zero contact angle assumption. 


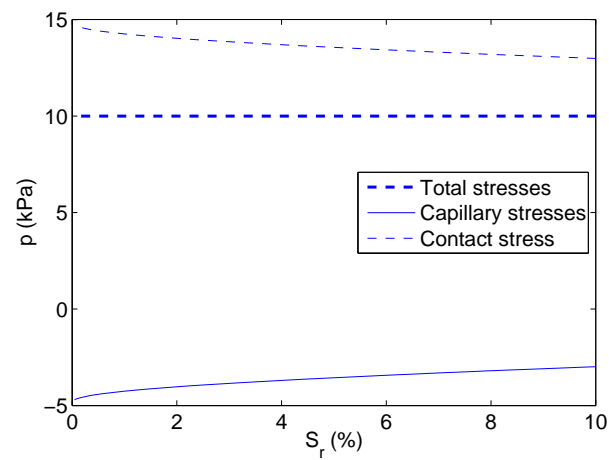

(a) Stresses

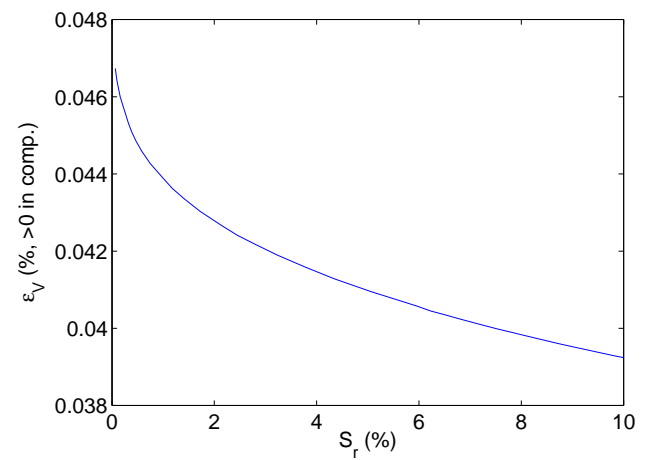

(b) Strain

Figure 6: Wetting of an isotropic sample

A significant capillary stress ( $p_{\text {cap }} \approx-5 \mathrm{kPa}$ for $S_{r} \rightarrow 0$ ) is developed at the drying-wetting transition (Fig. 6(a)) . Further wetting makes the capillary stress decrease (in absolute value) because of the decrease in suction that is not counterbalanced by the increase in wetted surfaces upon which $\chi$ depends. As for the strains, pure volumetric strains are induced during wetting of the isotropic sample. The drying-wetting transition causes the sample to contract due to the development of attractive internal forces. The volumetric response is thereafter dilatant upon further wetting (Fig. 6(b) $)$, because at the meniscus scale liquid bonding decreases in intensity when suction decreases for menisci between contacting particles [16].

This behaviour is qualitatively consistent with the trends of $p_{\text {cont }}=$ $p-p_{\text {cap }}$ (Fig. 6(a)), if an elastic behaviour were assumed between strains and the particle contact stress $\sigma^{\text {cont }}=\sigma-\sigma^{\text {cap }}$. For later reference, Section 7 investigates more carefully whether this stress tensor can be regarded as 


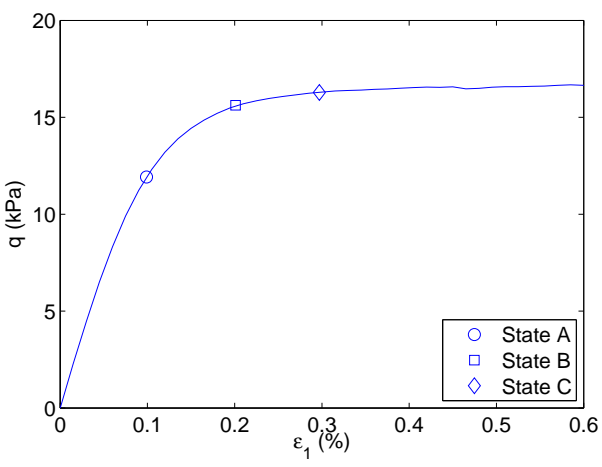

(a) $q\left(\varepsilon_{1}\right)$

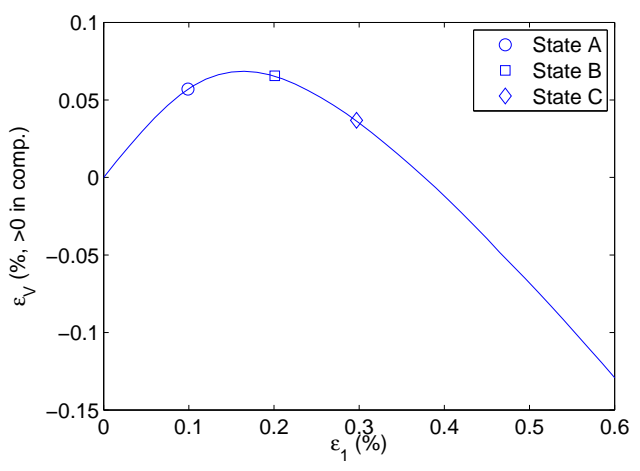

(b) $\varepsilon_{V}\left(\varepsilon_{1}\right)$

Figure 7: Initial dry axisymmetric compression defining States A, B and C

After an axial strain of $0.1 \%$, plastic deformations with particle rearrangement has not yet fully developed; thus State A is almost isotropic 

differences in SWCC are negligible between the three states (Fig 9).

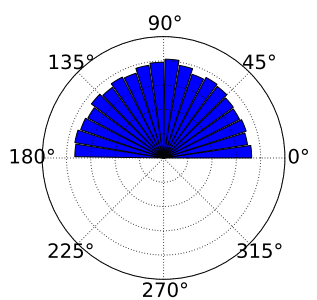

(a) State A

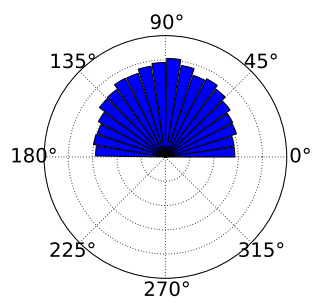

(b) State B

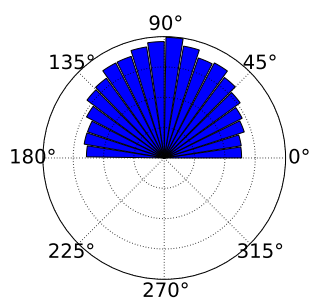

(c) State C and thus induced anisotropy appears clearly in Fig. 8(b) and 8(c), with the State $\mathrm{C}$ being more marked due to a greater plastic deformation. However,

Figure 8: Contact directions in a plane of symmetry ( $y$-direction corresponds to $90^{\circ}$ )

448

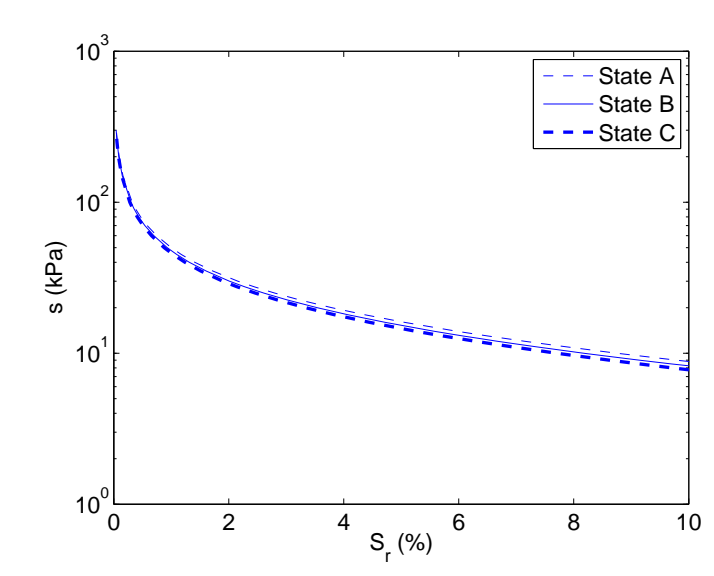

Figure 9: SWCC for the anisotropic states

(Fig. 8(a)). On the other hand, States B and C are close to the peak stress,

Although wetting is being imposed to the three different states under a constant total stress, a deviatoric component for the capillary stresses interestingly appears due to the induced anisotropy (Fig. 10(b)]. As such, the deviatoric capillary stress increases (in absolute value) from State A to 
State $C$, irrespective of the water content. Except for the dry-wet transition, variations in water content have generally little effect on the deviatoric capillary stress. Indeed, an increasing degree of saturation reduces the deviatoric component of $-s \mathcal{X}$ on one hand, and increases that of $-\boldsymbol{B}$ on the other hand (Fig. 11). An increase in the deviatoric component of $-\boldsymbol{B}$ (i.e. its norm) upon saturation corresponds to greater menisci contours along which surface tension forces act. On the other hand, the decrease in suction associated with saturation is responsible for decreasing the deviatoric part and the mean pressure (Fig. 10(a)) of $-s \chi$. Considering the different states, the mean capillary stress is observed to decrease (in absolute value) from State A to State $\mathrm{C}$. This comes from a decreasing coordination number between State A and C, creating here less liquid bridges.

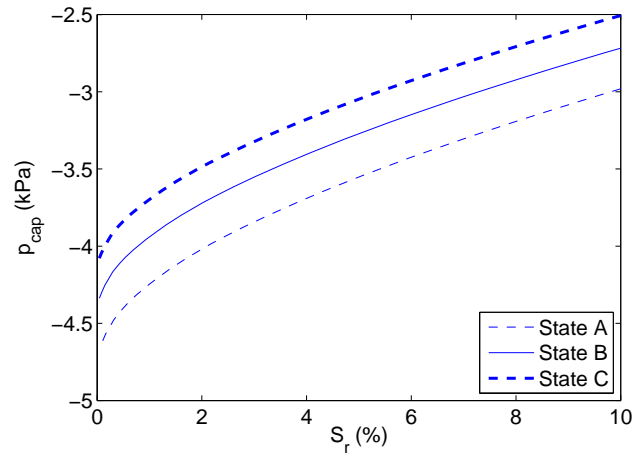

(a) Mean stress

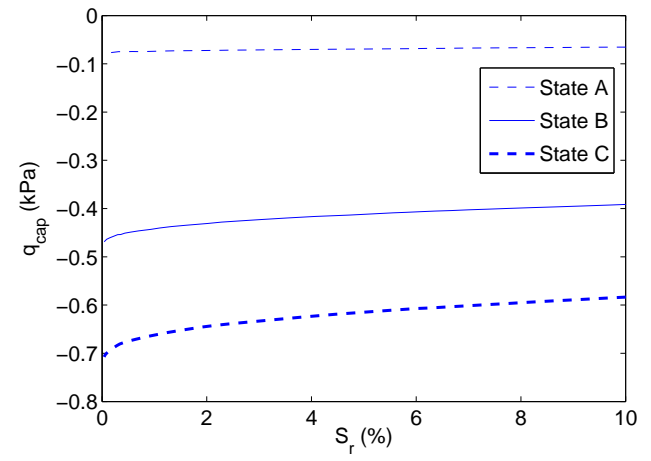

(b) Deviatoric stresses

Figure 10: Capillary stresses during wetting of anisotropic packings

As for the strains, the wetting of anisotropic assemblies clearly induces deviatoric strains, in addition to the volumetric ones. The deviatoric strain 


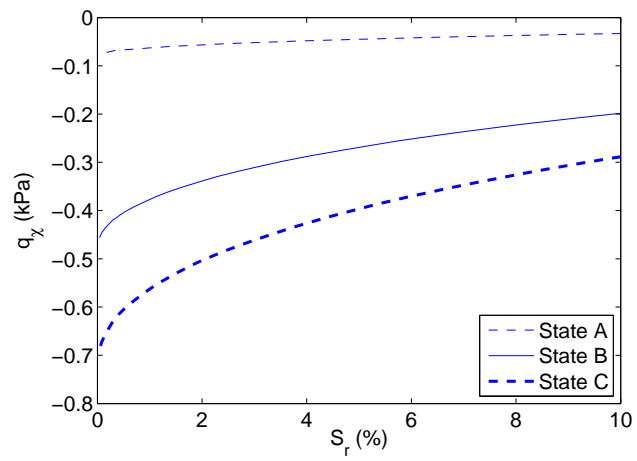

(a) $-s \chi$

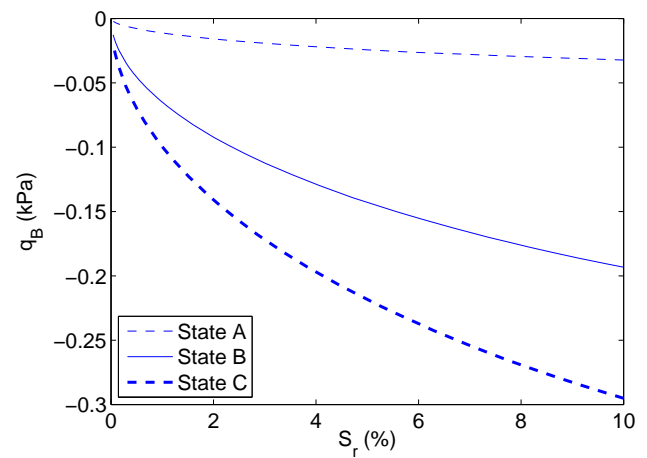

(b) $-B$

Figure 11: Deviatoric components of the two parts of the capillary stress tensor

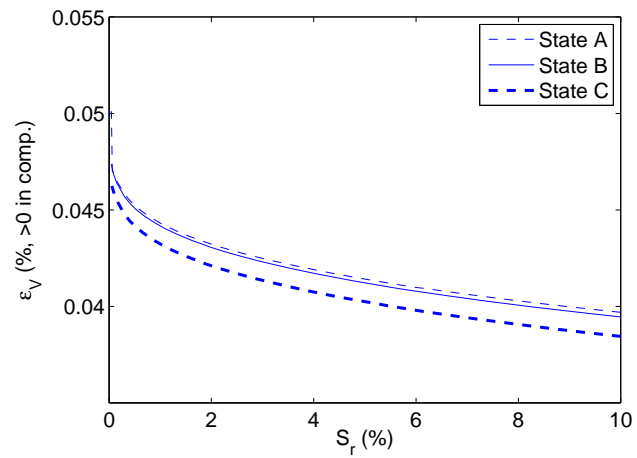

(a) Volumetric strain $\varepsilon_{V}$

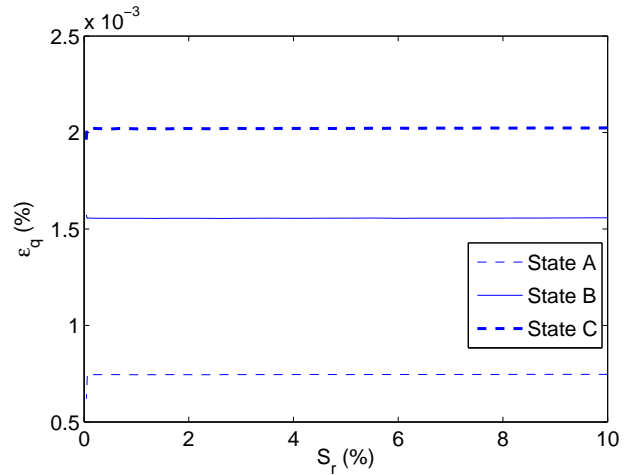

(b) Deviatoric strain $\varepsilon_{q}=2 / 3\left(\varepsilon_{y y}-\varepsilon_{\text {lat }}\right)$

Figure 12: Deformations (with respect to the dry states A, B or C) upon wetting of anisotropic packings 
Both volumetric and deviatoric strain changes are qualitatively consistent with the changes of the intra-granular stress $\sigma^{\text {cont }}=\Sigma-\sigma^{\text {cap }}$ (Fig. 13) but these results do show yet any quantitative comparison.

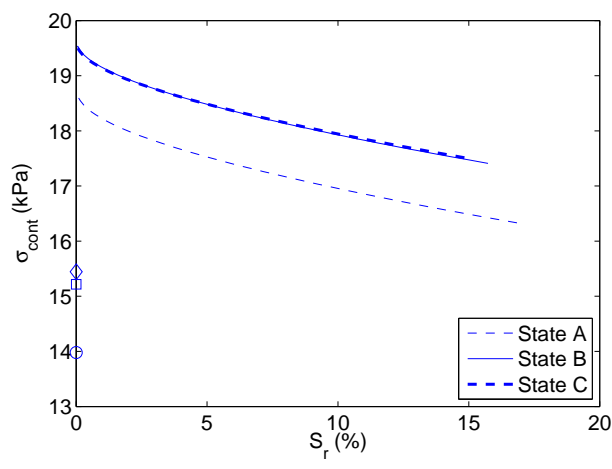

(a) Mean stress

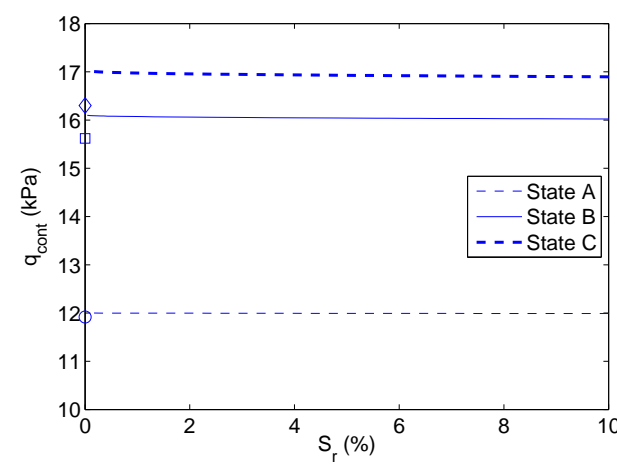

(b) Deviatoric stress

Figure 13: Intra-granular stress $\sigma^{\text {cont }}=\sigma-\sigma^{\text {cap }}$ upon wetting of anisotropic packings. Symbols correspond to $\sigma^{\text {cont }}$ in the dry case, after the initial dry compression. They correspond also to the constant total stresses during wetting

\section{Mechanical loading and anisotropy of the capillary stress tensor}

The capillary stress tensor $\sigma_{\text {hom }}^{\text {cap }}=-(s \boldsymbol{\chi}+\boldsymbol{B})$ is now computed using Eq. (12) along triaxial and simple shear loadings (see Fig. 14). The loading paths are applied to the discrete model for low degrees of saturation (Table 3), so that both approaches are quantitatively consistent: $\sigma_{\text {hom }}^{\text {cap }} \approx \sigma_{\text {DEM }}^{\text {cap }} \approx \sigma^{\text {cap }}$. Considering initial states resulting from a wetting path, menisci are created at geometrical contacts only. Hence, initial states show in average five menisci per particle. Then, menisci are kept when initially touching particles separate during loading, as long as Laplace's equation can be solved. Note that triaxial simulations involve frictionless 

[15].
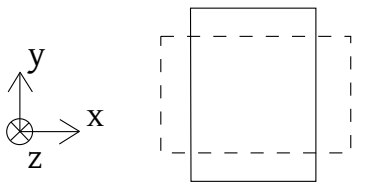

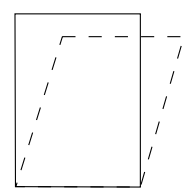

Figure 14: Triaxial loading (left): $\Sigma_{I}=\Sigma_{y y}>\Sigma_{I I}=\Sigma_{x x}=\Sigma_{I I I}=\Sigma_{z z}=\Sigma_{\text {lat }}=c s t$, and simple shear loading (right): $\partial v_{x} / \partial y=c s t, \Sigma_{y y}=\Sigma_{z z}=\Sigma_{l a t}=c s t$

Table 3: Considered mechanical loading paths

\begin{tabular}{c|c|c|c}
$\begin{array}{c}\text { Loading } \\
\text { type }\end{array}$ & $\begin{array}{c}\Sigma_{\text {lat }} \\
(\mathrm{kPa})\end{array}$ & $\begin{array}{c}S \\
(\mathrm{kPa})\end{array}$ & $\begin{array}{c}\text { Initial } S_{r} \\
(\%)\end{array}$ \\
\hline Triaxial & 10 & 20 & 3.78 \\
" & " & 50 & 1.00 \\
" & " & 100 & 0.32 \\
" & " & 300 & 0.05 \\
Simple shear & " & 50 & 1.16 \\
" & " & 300 & 0.05
\end{tabular}

rigid platens as boundary DE, while simple shear simulations use periodic boundaries. The periodic sample used for simple shear simulations is as close as possible to the non-periodic one used for triaxial simulations, see

$$
\text { simple shear loading (right). } v_{x} / \partial y=c s t, \Sigma_{y y}=\Sigma_{z z}=\Sigma_{l a t}=c s t
$$

We focus on the relative contributions of the terms $s \chi$ and $B$, and the anisotropy (deviatoric nature) of the capillary stress. Tensors are represented using three invariants: the mean stress $p$, the deviatoric stress $q=\Sigma_{y y}-\Sigma_{\text {lat }}$ (for triaxial loadings) or $|q|=\sqrt{3 / 2}\|\boldsymbol{s}\|=\sqrt{3 / 2}\|\boldsymbol{\Sigma}-p \delta\|$ (for simple shears), and the Lode angle $\theta=1 / 3 \operatorname{acos}\left(27 / 2 \operatorname{det}(s) /|q|^{3}\right)$. For instance, $\theta=0^{\circ}$ for triaxial compression. 


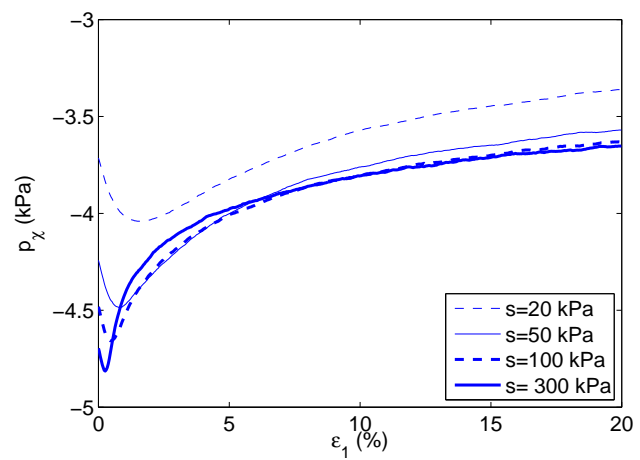

(a) Mean stress

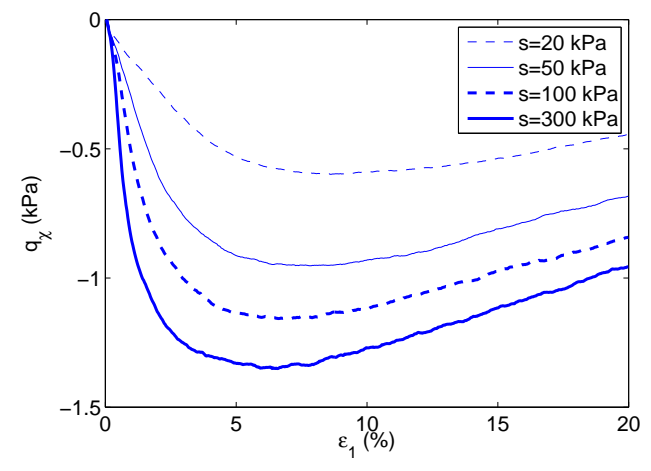

(b) Deviatoric stress

Figure 15: Invariants of $-s \chi$ for triaxial loadings with different suctions

As explained earlier, the initially isotropic packing makes the capillary stress to be spherical and equal to $-s \chi(B=0)$ at the start of the triaxial loading (Fig. 15 and 16). As the loading progresses and induced anisotropy develops, the deviatoric nature of the capillary stress tensor, as measured by $\left|q_{\text {cap }}\right| / p_{\text {cap }}$, rapidly builds up (Fig. 17). For low suctions this deviatoric nature arises from the tensor $-\boldsymbol{B}$ describing the spatial distribution of surface tension forces, whereas for high suctions it is the spatial distribution of matric suction $(-s \chi)$ term that governs. Indeed, for a given strain value, Fig. 15(b) and 16 confirm the influence of matric suction shown previously: the deviatoric part of $(-s \chi)$ increases according to suction, due to the proportionality with $s$, whereas the deviatoric tensor $B$ decreases in norm with suction because of dwindling wetted contours. Changes in the mean and deviatoric parts of the capillary stress with suction follow the same trends for simple shear loading [15]. Furthermore, 


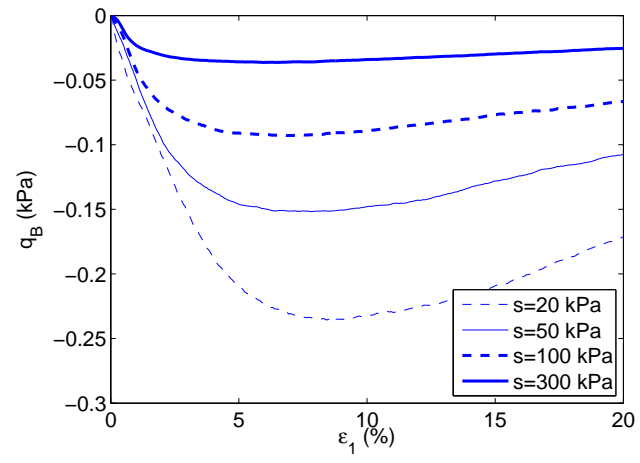

Figure 16: Deviatoric stress of $-\boldsymbol{B}$ for triaxial loadings with different suctions

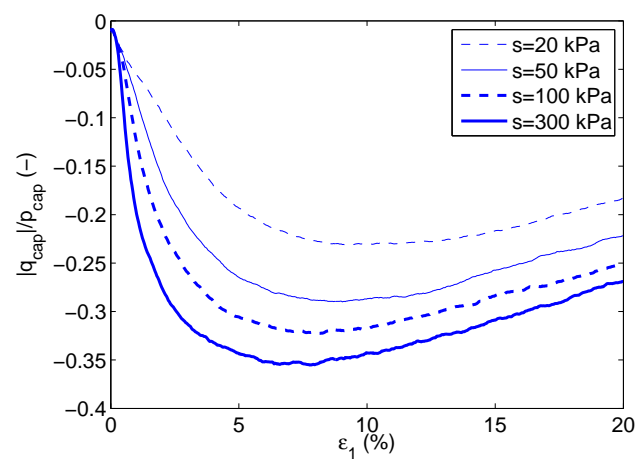

Figure 17: Deviatoric feature of $\sigma^{c a p}=-s \chi-B$ for triaxial loadings with different suctions

the mechanical actions described by tensors $-s \chi$ and $-B$ present the same Lode angles in simple shear (Fig. 18).

\section{Effective stress}

We finally focus our attention to the particle contact stress $\sigma^{\text {cont }}$ which is essentially an intra-granular stress for very low saturation ratios $S_{r}<$ $1 \%$ for which DEM is equivalent to the homogenization approach. The DEM model is used to impose various total stresses $\Sigma$ and compute the 


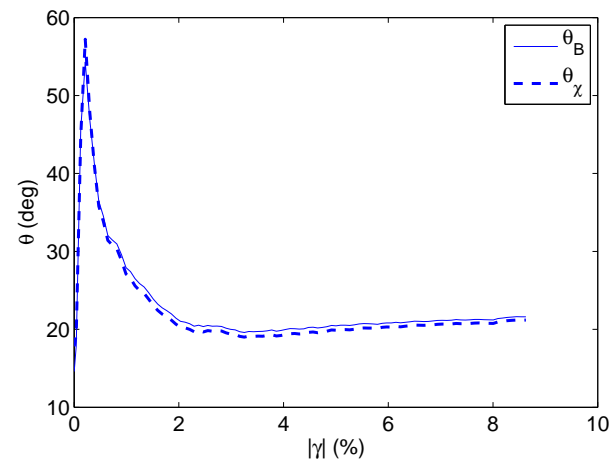

(a) $s=50 \mathrm{kPa}$

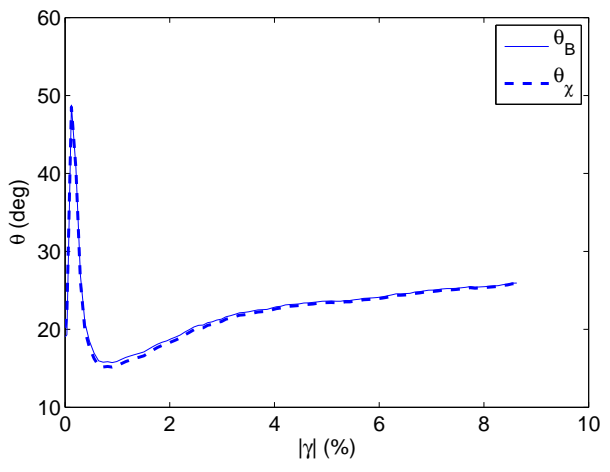

(b) $s=300 \mathrm{kPa}$

Figure 18: Lode angles of the two parts of the capillary stress tensor for simple shears

capillary stress $\sigma^{\text {cap }}=-s \chi-B$. The intra-granular stress is finally indirectly deduced from $\sigma^{\text {cont }}=\Sigma-\sigma^{\text {cap }}$.

\subsection{Constitutive relevancy of the intra-granular stress}

First, two triaxial loadings in dry and wet conditions are considered. A confining pressure close to $25 \mathrm{kPa}$ is applied in the dry test, whereas 20 $\mathrm{kPa}$ of confining pressure together with $300 \mathrm{kPa}$ of suction are imposed in the unsaturated case. This corresponds throughout the wet test to a degree of saturation $S_{r} \approx 0.04 \% \pm 0.01 \%$. The intra-granular stresses $\sigma^{\text {cont }}$ cannot be directly controlled during wet simulations and these loading parameters allow $\sigma^{\text {cont }}$ to be as close as possible during both simulations, see Fig. 19(a), It turns out that a significant discrepancy appears on the resulting strain paths as seen in Fig. 19(b), In fact, the stress tensor $\sigma^{\text {cont }}$ can arguably be considered as specific to the solid phase since it describes the stresses arising inside the solid grains due to other solid grains. How- 


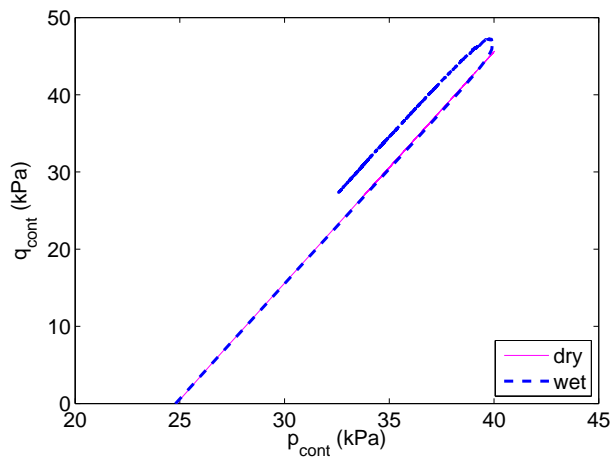

(a) Intra-granular stress path $q_{\text {cont }}\left(p_{\text {cont }}\right)$

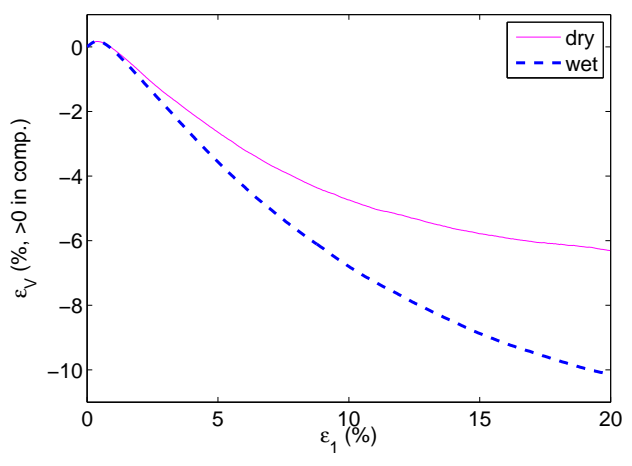

(b) $\varepsilon_{V}\left(\varepsilon_{1}\right)$

Figure 19: Comparable loading paths in dry and wet conditions

ever, as illustrated by our results, the straining of the solid phase inside the unsaturated REV (i.e. straining of the unsaturated REV itself) is affected by the behaviour of the other phases and also the coupling between the different phases [30]. For that reason, we observe different behaviours between the dry and wet cases.

It is probable that the observed strains of the unsaturated REV could still be interpreted using $\sigma^{\text {cont }}$ as a stress variable, through a phenomenological elasto-plastic approach including suction hardening [10].

\subsection{A unique plastic limit criterion}

In this last section, attention is paid to the attainment of limit plastic stress states starting from several triaxial and simple shear loading paths under both dry and wet conditions. Confining pressures range between 5 and $25 \mathrm{kPa}$ and, for the unsaturated cases, suction values from 50 to 600 $\mathrm{kPa}$; which corresponds to different degrees of saturation below $1 \%$. The 

strength of the DEM assembly in both dry and wet conditions. angle influence $f(\theta)$ is assumed to obey Lade criterion [24].

Whereas no unique plastic limit criterion appears for the total stresses in both dry and wet conditions as seen in Fig. 20(a), the use of $\sigma^{\text {cont }}$ makes all data points fit within an acceptable agreement into a unique plastic limit criterion corresponding (for triaxial compression) to a Mohr-Coulomb friction angle $\phi_{M C} \approx 29^{\circ}$ (Fig. 20(b)). This suggests that the intra-granular stress defined as $\sigma^{\text {cont }}=\Sigma-\sigma^{\text {cap }}$ is the adequate variable to express the

limit stress states are defined as the maxima of $(q f(\theta) / p)$, with the Lode

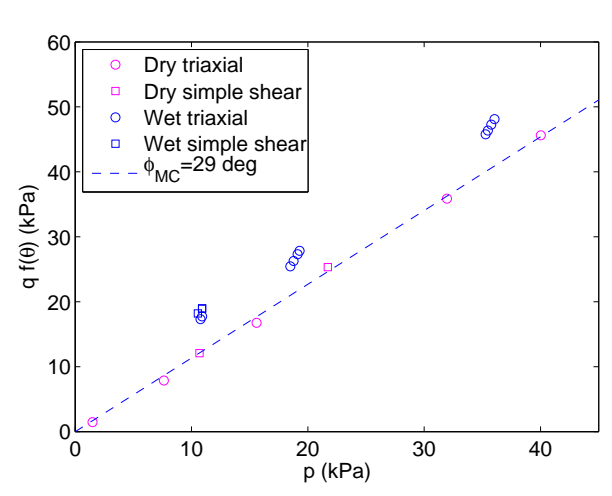

(a) Total stresses

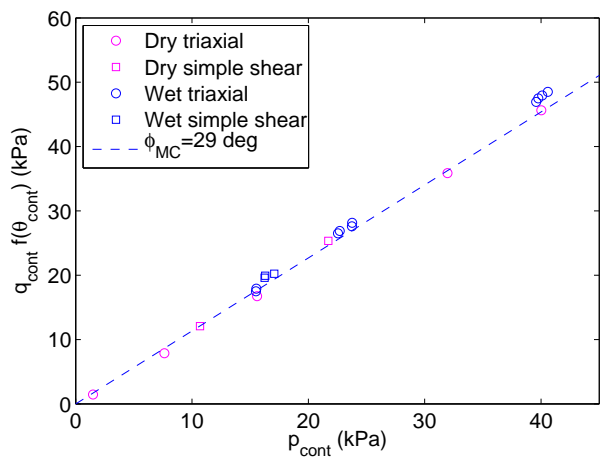

(b) Intra-granular stress $\sigma^{\text {cont }}$
Figure 20: Plastic limit criterion for the granular soil in dry and wet conditions

\section{Conclusion}

The microscopic stresses within an unsaturated pendular granular soil have been volume-averaged to give a tensorial form for the macroscopic stress. The averaging process considers in particular interfacial stress tensors specific for the discontinuity surfaces between air and water. Deriva- 
tions led to the expression of the total net stress tensor as the sum of a capillary stress tensor, describing mechanical actions pertaining to the liquid bridges and interface surfaces, and a contact stress tensor, encompassing the contact forces between solid grains.

These different stress tensors have been evaluated and verified using a DEM model for unsaturated granular soils. A major finding is that comparisons between the DEM and the homogenization approach is strictly speaking valid only for very low degrees of saturation, at the low end of the pendular regime. For higher water content, internal forces such as water pressure and surface tension forces act on finite surfaces, whereas DEM can only consider resultant point forces, which leads to an inconsistent description of total stresses for the unsaturated REV.

Using then the DEM model in conjunction with the averaging procedure for very low water content, the capillary stresses have been shown to be anisotropic in the general case. Non-spherical stress tensors are thus required to describe these capillary stresses, in contradiction with the classical Bishop's equation and current modelling approaches.

Furthermore, the effective nature of the particle contact stress tensor has been discussed. From a theoretical standpoint, this stress tensor cannot be used to assess deformations in an unsaturated sample in the same manner as for a dry one. However, results from triaxial and simple shear loading paths point to show the particle contact stress tensor does unify the description of failure in unsaturated and dry granular soils. Further 
investigations of homogenization approaches wherein strains are also introduced along with the consideration of interfaces and compressibility of phases should provide further answers to open questions as to the existence of a single-valued stress tensor that controls deformations in unsaturated granular assemblies.

\section{Acknowledgements}

This work is supported by the Natural Science and Engineering Research Council of Canada and Foundation Computer Modelling Group within the framework of a Government-Industry Partnership (NSERCCRD) Grant towards the fundamental understanding of complex multiphasic granular media.

\section{Appendix A.}

Recall Stokes's theorem:

$$
\int_{\mathcal{C}} \mathbf{F} \cdot d \boldsymbol{\ell}=\int_{S} \mathbf{n} \cdot(\nabla \times \mathbf{F}) d S
$$

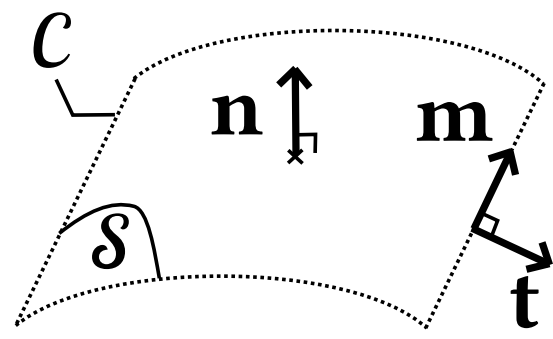

Figure A.1: Definition of surface $S$, contour $\mathcal{C}$ and associated vectors 
Let $\mathbf{F}=\mathbf{f} \times \mathbf{b}$ and $d \boldsymbol{\ell}=\mathbf{m} d \boldsymbol{\ell}$ where $\mathbf{b}$ is a constant vector and $\mathbf{m}$ the unit vector tangent to contour $\mathcal{C}$. Then,

$$
\int_{\mathcal{C}}(\mathbf{f} \times \mathbf{b}) \cdot \mathbf{m} d \ell=\int_{S} \mathbf{n} \cdot(\nabla \times(\mathbf{f} \times \mathbf{b})) d S
$$

Using identities

$$
\begin{aligned}
(\mathbf{f} \times \mathbf{b}) \cdot \mathbf{m} & =-\mathbf{b} \cdot(\mathbf{f} \times \mathbf{m}) \\
\nabla \times(\mathbf{f} \times \mathbf{b}) & =-\mathbf{b}(\nabla \cdot \mathbf{f})+\mathbf{b} \cdot \nabla \mathbf{f}
\end{aligned}
$$

and since $\mathbf{b}$ is an arbitrary vector,

$$
\int_{\mathcal{C}}(\mathbf{f} \times \mathbf{m}) d \ell=\int_{S} \mathbf{n}(\nabla \cdot \mathbf{f})-(\nabla \mathbf{f}) \cdot \mathbf{n} d S
$$

If we choose $\mathbf{f}=\gamma \mathbf{n}$ and recalling that $\mathbf{n} \times \mathbf{m}=-\mathbf{t}$

$$
\begin{aligned}
-\int_{\mathcal{C}} \gamma \mathbf{t} d \ell & =\int_{S} \mathbf{n}(\nabla \cdot(\gamma \mathbf{n})-(\nabla \gamma \mathbf{n}) \cdot \mathbf{n} d S \\
& =\int_{S}[\mathbf{n} \nabla \gamma \cdot \mathbf{n}+\gamma \mathbf{n}(\nabla \cdot \mathbf{n})-\nabla \gamma-\gamma(\nabla \mathbf{n}) \cdot \mathbf{n}] d S
\end{aligned}
$$

Since $\nabla \gamma$ is tangent to surface $S$ and $(\nabla \mathbf{n}) \cdot \mathbf{n}=\frac{1}{2} \nabla(\mathbf{n} \cdot \mathbf{n})=\frac{1}{2} \nabla(1)=0$, we finally get:

$$
\int_{\mathcal{C}} \gamma \mathbf{t} d \ell=\int_{S}[\nabla \gamma-\gamma \mathbf{n}(\nabla \cdot \mathbf{n})] d S
$$

\section{References}

\section{References}

[1] Alonso, E. E., Gens, A., Josa, A., 1990. A constitutive model for partially saturated soils. Géotechnique 40, 405-430(25). 
URLhttp://www.icevirtuallibrary.com/content/article/10.1680/geot.1990.40.3.4

[2] Bagi, K., 1996. Stress and strain in granular assemblies. Mechanics of Materials 22 (3), $165-177$.

[3] Bathrust, R. J., Rothenburg, L., 1990. Observations on stress-forcefabric relationships in idealized granular materials. J. Mechanics of materials 9, 65-80.

[4] Biarez, J., Fleureau, J., Taibi, S., 1993. Constitutive model for unsaturated granular media made up by spheres. In: Thornton, C. (Ed.), Proc. 2nd Int. Conference on Micromechanics of Granular Media. Balkema, Birmingham, UK, pp. 51-58.

[5] Bishop, A., 1959. The principle of effective stress. Teknisk Ukeblad 39, 859-863.

[6] Bishop, A. W., Blight, G. E., 1963. Some aspects of effective stress in saturated and partly saturated soils. Géotechnique 13, 177-197.

[7] Blight, G., 1965. A study of effective stress for volume change. In: Aitchison, G. (Ed.), Moisture Equilibria and Moisture Changes in Soils beneath Covered Areas. Butterworths, pp. 259-269.

[8] Bluhm, J., de Boer, R., 1996. Effective stresses - a clarification. Archive of Applied Mechanics 66 (7), 479-492.

URL http://dx.doi .org/10.1007/BF00790180 
[9] Borja, R. I., 2004. Cam-clay plasticity. part v: A mathematical framework for three-phase deformation and strain localization analyses of partially saturated porous media. Computer Methods in Applied Mechanics and Engineering 193 (48-51), 5301 - 5338, advances in Computational Plasticity.

URL http://www.sciencedirect.com/science/article/pii/S0045782504002762

[10] Borja, R. I., 2006. On the mechanical energy and effective stress in saturated and unsaturated porous continua. International Journal of Solids and Structures 43 (6), $1764-1786$.

[11] Catalano, E., Chareyre, B., Barthélémy, E., 2014. Pore-scale modeling of fluid-particles interaction and emerging poromechanical effects. International Journal for Numerical and Analytical Methods in Geomechanics $38(1), 51-71$.

[12] Chareyre, B., Cortis, A., Catalano, E., Barthélemy, E., 2012. Pore-scale modeling of viscous flow and induced forces in dense sphere packings. Transport in Porous Media 94 (2), 595-615.

[13] Chateau, X., Dormieux, L., 2002. Micromechanics of saturated and unsaturated porous media. International Journal for Numerical and Analytical Methods in Geomechanics 26 (8), 831-844.

[14] Cundall, P., Strack, O., 1979. A discrete numerical model for granular assemblies. Géotechnique 29, 47-65. 
[15] Duriez, J., Wan, R., 2015a. Effective stress in unsaturated granular materials: micro-mechanical insights. In: Coupled Problems in Science and Engineering VI. pp. 1232-1242.

[16] Duriez, J., Wan, R., 2015b. Micromechanics of wet granular soils and issues in discrete element modelling. Géotechnique - under review.

[17] Fredlund, D., Morgenstern, N., 1977. Stress state variables for unsaturated soils. Journal of Geotechnical and Geoenvironmental Engineering 103 (GT5), 447-466.

[18] Gray, W. G., Hassanizadeh, S. M., 1991. Unsaturated flow theory including interfacial phenomena. Water Resources Research 27 (8), 1855-1863.

[19] Gray, W. G., Schrefler, B. A., Pesavento, F., 2009. The solid phase stress tensor in porous media mechanics and the hill-mandel condition. Journal of the Mechanics and Physics of Solids 57 (3), 539 - 554.

URL http://wWw.sciencedirect.com/science/article/pii/S0022509608001968

[20] Hassanizadeh, S., Gray, W. G., 1990. Mechanics and thermodynamics of multiphase flow in porous media including interphase boundaries. Advances in Water Resources 13 (4), 169 - 186.

URL http://www.sciencedirect.com/science/article/pii/030917089090040B

[21] Houlsby, G. T., 1997. The work input to an unsaturated granular 
material. Géotechnique 47, 193-196(3).

URL http://www.icevirtuallibrary.com/content/article/10.1680/geot.1997.47.1.1

[22] Huang, X., O’Sullivan, C., Hanley, K., Kwok, C., 2014. Discreteelement method analysis of the state parameter. Géotechnique 64, 954-965(11).

[23] Jennings, J. E. B., Burland, J. B., 1962. Limitations to the use of effective stresses in partly saturated soils. Géotechnique 12, 125-144(19). URL http://www. icevirtuallibrary.com/content/article/10.1680/geot.1962.12.2.1

[24] Lade, P. V., Duncan, J. M., 1975. Elasto-plastic stress-strain theory for cohesionless soil. Journal of the Geotechnical Engineering Division $101(10)$, 1037-1053.

[25] Laplace, P. S., 1806. Sur l'action capillaire in Supplément au Livre X du Traité de Mécanique Céleste. Duprat (Paris), available in Oeuvres complètes de Laplace, Tome 4, on www.gallica.bnf.fr.

[26] Li, X., 2003. Effective stress in unsaturated soils: a microstructural analysis. Géotechnique 53 (2), 273-277.

[27] Likos, W. J., 2014. Effective stress in unsaturated soil: Accounting for surface tension and interfacial area. Vadose Zone Journal 13 (5), 1-12.

[28] Love, A., 1927. A treatise on the mathematical theory of elasticity. Cambridge University Press, Cambridge. 
[29] Lu, L., Likos, W., 2004. Unsaturated Soil Mechanics. John Wiley.

[30] Madeo, A., dell'Isola, F., Darve, F., 2013. A continuum model for deformable, second gradient porous media partially saturated with compressible fluids. Journal of the Mechanics and Physics of Solids 61 (11), $2196-2211$.

URL/http://www.sciencedirect.com/science/article/pii/S002250961300121X

[31] Mani, R., Kadau, D., Herrmann, H. J., 2013. Liquid migration in sheared unsaturated granular media. Granular Matter 15 (4), 447-454.

[32] Nuth, M., Laloui, L., 2008. Effective stress concept in unsaturated soils: Clarification and validation of a unified framework. International Journal for Numerical and Analytical Methods in Geomechanics 32 (7), 771-801.

[33] Pietruszczak, S., Pande, G., 1991. On the mechanics of partially saturated soils. Computers and Geotechnics 12 (1), 55-71.

[34] Potyondy, D., Cundall, P., 2004. A bonded-particle model for rock. International Journal of Rock Mechanics and Mining Sciences 41 (8), $1329-1364$.

[35] Richefeu, V., E. Y. M. S., Radjaï, F., 2006. Shear strength properties of wet granular materials. Phys. Rev. E 73, 051304-1 -051304-11.

[36] Rosenkilde, C. E., 1967. Surface-energy tensors. Journal of Mathematical Physics 8 (1). 
[37] Roux, J.-N., Chevoir, F., 2005. Discrete numerical simulation and the mechanical behavior of granular materials. Bulletin des laboratoires des ponts et chaussées 254, 109-138.

[38] Scholtès, L., Chareyre, B., Nicot, F., Darve, F., 2009. Micromechanics of granular materials with capillary effects. International Journal of Engineering Science 47 (1), $64-75$.

[39] Scholtès, L., Hicher, P.-Y., Nicot, F., Chareyre, B., Darve, F., 2009. On the capillary stress tensor in wet granular materials. International Journal for Numerical and Analytical Methods in Geomechanics 33 (10), 1289-1313.

[40] Schrefler, B., 1995. F.e. in environmental engineering: Coupled thermo-hydro-mechanical processes in porous media including pollutant transport. Archives of Computational Methods in Engineering $2(3), 1-54$.

URL http://dx.doi.org/10.1007/BF02736173

[41] Skempton, A., 1961. Effective stress in soils, concrete and rock. In: In: Pore Pressure and Suction in Soils. Butterworth, pp. 4-16.

[42] Vlahinić, I., M.J., H., Andrade, J., Thomas, J., 2011. A novel and general form of effective stress in a partially saturated porous material: The influence of microstructure. Mechanics of Materials 43 (1), 25 - 
35.

URL http://www.sciencedirect.com/science/article/pii/S0167663610001547

[43] Šmilauer, V., Catalano, E., Chareyre, B., Dorofeenko, S., Duriez, J., Gladky, A., Kozicki, J., Modenese, C., Scholtès, L., Sibille, L., Stránský, J., Thoeni, K., 2010. Yade Documentation, 1st Edition. The Yade Project, http: //yade-dem.org.

[44] Wan, R., Khosravani, S., Pouragha, M., 2014. Micromechanical analysis of force transport in wet granular soils. Vadose Zone Journal 13 (5). URL http://vzj.geoscienceworld.org/content/13/5/vzj2013.06.0113.abstract

[45] Weber, J., 1966. Recherches concernant les contraintes intergranulaires dans les milieux pulvérulents. Bulletin de liaison des Ponts et Chaussées 20, 1-20. 